\title{
Performance of Cpred/Cobs concentration ratios as a metric reflecting adherence to antidepressant drug therapy
}

\author{
This article was published in the following Dove Press journal: \\ Neuropsychiatric Disease and Treatment \\ 16 March 2011 \\ Number of times this article has been viewed
}

\author{
Yan Feng' \\ Marc R Gastonguay ${ }^{2}$ \\ Bruce G Pollock ${ }^{3,5}$ \\ Ellen Frank ${ }^{3}$ \\ Gail H Kepple ${ }^{4}$ \\ Robert R Bies $5,6,7$ \\ 'Discovery Medicine and Clinical \\ Pharmacology, Bristol-Myers Squibb, \\ Lawrenceville, NJ, USA; ${ }^{2}$ Metrum \\ Institute, Tariffville, CT, USA; \\ ${ }^{3}$ Department of Psychiatry, School of \\ Medicine, ${ }^{4}$ Department of Depression \\ Prevention, University of Pittsburgh, \\ PA, USA; ${ }^{5}$ Centre for Addiction and \\ Mental Health, University of Toronto, \\ Toronto, Canada; ${ }^{6}$ Division of Clinical \\ Pharmacology, School of Medicine and \\ Center for Computational Biology \\ and Bioinformatics, Indiana University \\ School of Medicine, Indianapolis, \\ IN, USA; ${ }^{7}$ Indiana Clinical Translational \\ Research Institute, Indiana University \\ School of Medicine, IN, USA
}

Correspondence: Robert R Bies

Division of Clinical Pharmacology,

Center for Computational Biology and

Bioinformatics, Indiana University School of Medicine, I00I W I0th Street W7I38, Indianapolis, IN 46202, USA

$\mathrm{Tel}+\mathrm{I} 3176307868$

Fax +I 3176308185

Email rrbies@iupui.edu
Background: Nonadherence is very common among subjects undergoing pharmacotherapy for schizophrenia and depression. This study aimed to evaluate the performance of the ratio of the nonlinear mixed effects pharmacokinetic model predicted concentration to observed drug concentration (ratio of population predicted to observed concentration (Cpred/Cobs) and ratio of individual predicted to observed concentration (Cipred/Cobs) as a measure of erratic drug exposure, driven primarily by variable execution of the dosage regimen and unknown true dosage history.

Methods: Modeling and simulation approaches in conjunction with dosage history information from the Medication Event Monitoring System (MEMS, provided by the "Depression: The search for treatment relevant phenotypes" study), was applied to evaluate the consistency of exposure via simulation studies with scenarios representing a long half-life drug (escitalopram). Adherence rates were calculated based on the percentage of the prescribed doses actually taken correctly during the treatment window of interest. The association between Cpred/Cobs, Cipred/Cobs ratio, and adherence rate was evaluated under various assumptions of known dosing history.

Results: Simulations for those scenarios representing a known dosing history were generated from historical MEMS data. Simulations of a long half-life drug exhibited a trend for overprediction of concentrations in patients with a low percentage of doses taken and underprediction of concentrations in patients taking more than their prescribed number of doses. Overall, the ratios did not predict adherence well, except when the true adherence rates were extremely high (greater than $100 \%$ of prescribed doses) or extremely low (complete nonadherence). In general, the Cipred/Cobs ratio was a better predictor of adherence rate than the Cpred/Cobs ratio. Correct predictions of extreme (high, low) 7-day adherence rates using Cipred/Cobs were $73.8 \%$ and $64.0 \%$.

Conclusion: This simulation study demonstrated the limitations of the Cpred/obs and Cipred/obs ratios as metrics for actual dosage intake history, and identified that use of MEMS dosing history monitoring combined with sparse pharmacokinetic sampling is a more reliable approach.

Keywords: adherence, Medication Event Monitoring System, dosing history, modeling and simulation

\section{Introduction}

Adherence to drug therapy is a largely undermeasured and underappreciated phenomenon, which may possibly contribute to significant problems with response to therapy and toxicity. Nonadherence is very common among subjects receiving treatment for schizophrenia or depression, and potentially contributing to substantial variability in response. ${ }^{1-3}$ Researchers have demonstrated that poorly adherent subjects with schizophrenia had a higher risk of rehospitalization. ${ }^{1}$ In addition, the relationship 
of the time to first drug holiday with fluoxetine treatment and the probability of response among subjects with major depressive disorder were related. ${ }^{3}$ Therefore, measuring an individual's specific dosage history condition is a very important piece of information in clinical trials, because the nonresponse or adverse drug effect may be caused by inconsistent drug intake.

The major measures of adherence include electronic monitoring of a dosage form container (eg, the Medication Event Monitoring System [MEMS] $]^{4}$ caps, ie, the gold standard), pill count, patient interview/rating scales, pharmacokinetic indicators, and the evaluation of prescription refill records. ${ }^{5-8}$ All of these methodologies are indirect approaches and have significant limitations.

A poor understanding of the adherence pattern that is most likely to contribute to a therapeutic problem ${ }^{3}$ represents a significant challenge in the area of pharmacotherapy. This issue is of particular importance in psychiatry where individuals with depression may be particularly susceptible to adherence patterns that relate to drug holidays as opposed to erratic, albeit consistent intake. ${ }^{3}$

Adherence can be conceptualized as the course of drug treatment and comprises three major components. These comprise acceptance of drug therapy (also referred to as initiation of drug therapy, ie, the patient starts taking the medication), execution of the prescribed drug regimen (ie, comparing the two time courses of drug intake, actual versus ideal), and discontinuation of the drug regimen (ie, the patient decides to stop taking the medication altogether). Acceptance and discontinuation are dichotomous, whereas execution is continuous. Persistence on drug therapy is defined as the time between acceptance and discontinuation. ${ }^{9}$ The method we propose for capturing erratic adherence patterns relates solely to the execution of the drug regimen.

Vrijens et $\mathrm{al}^{10,11}$ reported that using the detailed records of subjects' dosing history not only helped to achieve convergence in model fitting under sparse sampling measurement situations, but also explained $40 \%$ of residual variability in plasma lopinavir concentrations and reduced overall variability by $55 \%$. Utilization of a prior established pharmacokinetic/pharmacodynamic model may allow one to exploit this variability by evaluating the deviation between the prior model predicted and the observed drug concentrations at the population level (Cpred/Cobs), as well as the individual (Cipred/Cobs) level.

The primary objective of this study was to assess the stability and robustness of the ratio of Cpred/Cobs and Cipred/Cobs in predicting adherence rates. This was accomplished using simulation approaches that incorporate design features of a typical psychiatric clinical trial (Depression: The search for treatment relevant phenotypes. see http:/clinicaltrials.gov/ct/ show/NCT00073697) ${ }^{12}$ using the pharmacokinetic characteristics of escitalopram as a representative long half-life drug. We hypothesize that the magnitude of the ratios of Cpred/ Cobs and/or Cipred/Cobs predict adherence (ie, percentage of doses taken in a time window of interest).

\section{Methods}

\section{Adherence definition and categorization}

MEMS caps records from the study sponsored by the National Institute of Mental Health entitled "Depression: The search for treatment relevant phenotypes" 12 were used to supply the "true" dosage histories. Determination of the adherence patterns used the MEMS patterns divided into two different observation windows ( 2 days and 7 days) immediately prior to the sampling time. The adherence "rate" was calculated from the dosage history obtained from MEMS caps into a proportion of dosages correctly taken over the window period of interest.

Specifically, adherence rate was equal to the total number of MEMS caps openings recorded divided by the number of doses prescribed. Adherence rates were categorized according to total number of dosing events and frequency of drug administration (Table 1). The adherence rate was calculated for both 2-day and 7-day windows for each simulated clinic visit. This rate was used to classify adherence into percentage categories.

\section{Trial simulation}

Observed concentrations were generated by the simulation of the virtual patients (ie, Cobs). The predicted concentrations were obtained using NONMEM estimation steps (the population predicted and individual predicted represents the model predicted concentration at that sampling time based on the population prediction [Cpred] as well as the individual

Table I Categorization of 7-day and 2-day adherence rate

\begin{tabular}{lll}
\hline Group & 7-day history & 2-day history \\
\hline 1 & $>100 \%$ & $>100 \%$ \\
2 & $85 \%-100 \%$ & $50 \%-100 \%$ \\
3 & $30 \%-85 \%$ & $0 \%-50 \%$ \\
4 & $0 \%-30 \%$ & $0 \%$ \\
5 & $0 \%$ & $\mathrm{NA}$ \\
\hline
\end{tabular}

Notes: Adherence rates were categorized given total number of dosing events and frequency of drug administration. Seven-day and 2-day history categories differ due to different numbers of dosage events possible in each time window allowing additional resolution in the 7-day history group.

Abbreviation: NA, not available. 
prediction [Cipred]). A diagram depicting the simulation and estimation steps is shown in Figure 1.

\section{Analysis platform}

Nonlinear mixed effects modeling was employed for the population pharmacokinetic analysis as implemented in the NONMEM computer program (Version V, Icon Development Solutions, Elicott City, MD). ${ }^{13,14}$ The models consisted of a structural model that described the disposition of the drug following oral administration, and a pharmacostatistical model that described the residual interindividual and intraindividual variability. NONMEM was also utilized for performing the model-based simulations. "Virtual subjects" datasets simulation, graphics, and postprocessing of NONMEM outputs were performed using S-PLUS (Version 6.2.1; Insightful, Seattle, WA). Perl (version.5.6) was utilized as a scripting language to assist in automation of data extraction and simulation routines.

\section{Pharmacokinetic model for escitalopram}

An established escitalopram model was utilized to represent the long half-life drug. ${ }^{15,16}$ A two-compartment model with additive and proportional residual error models was adapted from the literature reports for escitalopram as a long half-life drug (Table 2). The models were then used to generate a unique set of pharmacokinetic parameters for each patient using the

Step 1: MEMS cap data - Generation of "virtual subject" dosing history

KNOWN DOSAGE HISTORY (KDH)

Step 3: Estimation of pharmacokinetic profile under known dosing history scenario (MEMS + actual sampling time)

Step 2: Simulation to generate pharmacokinetic profile using: Known dosing history (MEMS)+ simulated pharmacokinetic sampling time at each clinic visit (actual sampling time) and pharmacokinetic model

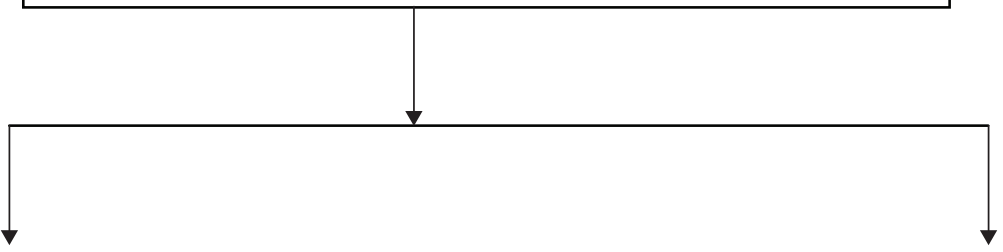

UKNOWN DOSAGE HISTORY (UDH)

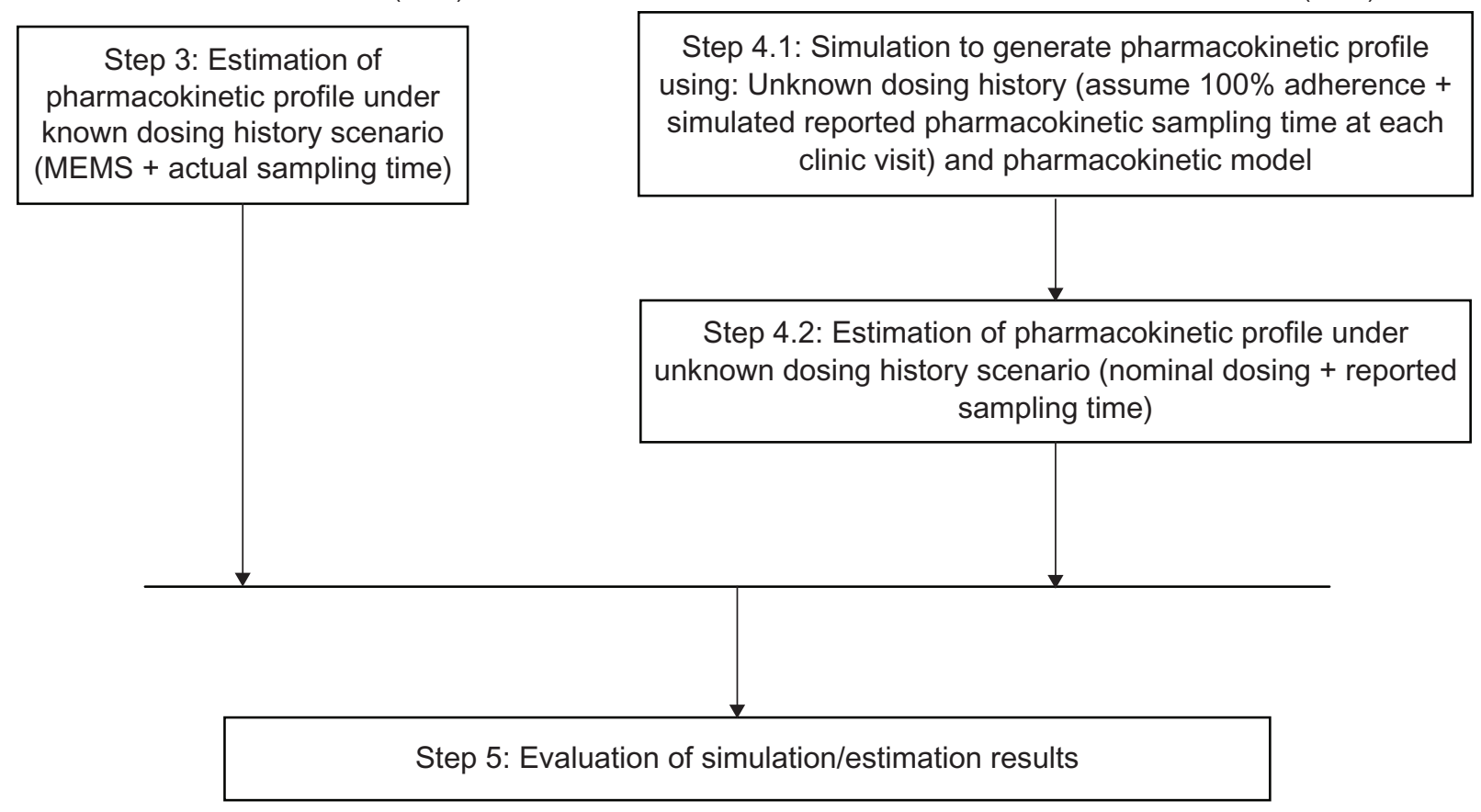

Figure I Flow chart of simulation and estimation approach under known dosage history and unknown dosage history scenarios. Abbreviation: MEMS, medication event monitoring system. 
Table 2 Pharmacokinetic parameters for a long half-life drug

\begin{tabular}{ll}
\hline Parameters & $\begin{array}{l}\text { Parameter estimates } \\
\text { for long half-life drug }\end{array}$ \\
\hline $\mathrm{CL}(\mathrm{L} / \mathrm{h})$ & 24.5 \\
$\mathrm{~V} 2(\mathrm{~L})$ & 417 \\
$\mathrm{Q}(\mathrm{L} / \mathrm{h})$ & 35.7 \\
$\mathrm{~V} 3(\mathrm{~L})$ & 541 \\
$\mathrm{Ka}(/ \mathrm{h})$ & 0.16 \\
$\omega_{\mathrm{CL}} \%$ & 50 \\
$\omega_{\mathrm{v2}} \%$ & 35 \\
$\omega_{\mathrm{Q}} \%$ & 30 \\
$\omega_{\mathrm{v3}} \%$ & 30 \\
$\sigma 1 \%$ & 30 \\
\hline
\end{tabular}

Abbreviations: $\mathrm{CL}$, clearance; $\mathrm{V} 2$, volume of distribution of central compartment; V3, volume of distribution of peripheral compartment; Q, intercompartment clearance; $\omega$, coefficient of variation of interindividual variability; $\sigma$, coefficient of variation of residual error.

NONMEM program (two-compartment, ADVAN4 TRANS4). In the model component, the individual parameter estimates are modeled as a function of typical values for the population and individual random deviations. The interindividual variability of the pharmacokinetic parameters is described below:

$$
C L=T V C L \times \exp \left(\eta_{C L}\right)
$$

where TVCL is the typical value of clearance for the population, $\mathrm{CL}$ is the individual parameter estimate, and $\eta_{C L}$ is the interindividual variability term on CL, representing the difference between the individual parameter estimate and the population mean. The inter-individual random variability are assumed to be log-normally distributed, with a mean of zero and variance of $\omega^{2}$.

\section{Generation of Cobs, Cpred, and Cipred}

A simulated (observed) plasma drug concentration (Cobs) was compared with model predicted concentrations using population level (Cpred) and individual (Cipred) level parameter estimates. This was accomplished by fitting this simulated (observed) plasma drug concentration (Cobs) to generate the population and individual predicted concentration values. This is shown in the equation below:

$$
C_{o b s[i, j]}=f\left(x_{[i, j]}, \theta, \eta_{[i]}\right)+\varepsilon_{[i, j]}
$$

where $C_{o b s[i, j]}$ is the jth concentration measured in the ith subject, $X_{[i, j]}$ is the jth time in the ith subject, $\theta$ are the fixed effects parameters in the model, $\eta_{[i]}$ is the ith individual's deviation in fixed effect from the population estimate and $\varepsilon_{[i, j]}$ is the residual error associated with the jth measured concentration in the ith individual. The $C_{p r e d[i . j]}$ is evaluated at $\eta_{[i]}=0$ and $C_{\text {ipred }[i . j]}$ is evaluated at $\eta_{[i]}$ equal to the maximum a posteriori Bayes estimate of the individual random effect $\left(\eta_{[i]}\right)$. Each simulated clinic visit was associated with a single concentration measurement.

\section{Trial simulation and estimation}

A flow chart of simulation and estimation steps is presented in Figure 1. Simulation scenarios for the virtual trials are summarized in detail (Table 3), including number of subjects, pharmacokinetic sampling per subject, simulation replicates, etc. Figure one shows how the MEMS data (Step 1) were utilized as the actual dosing history (dose and time of dose taken) for subjects in the clinical trial "Depression: The search for treatment relevant phenotypes".

Subjects recruited in the clinical trial had chronic psychiatric disorders. Dosing histories for the simulated trials were obtained by bootstrap resampling from the actual MEMS database in the phenotypes study.

Simulation (Step 2) was used to generate the "observed" concentrations (Cobs) for subjects at each clinic visit using the NONMEM simulation option.

Table 3 Detailed description of simulation scenarios for a long

\begin{tabular}{|c|c|}
\hline Simulation profile & Simulation scenarios \\
\hline $\begin{array}{l}\text { Sample size for each } \\
\text { simulation replicate }\end{array}$ & 65 \\
\hline Dose (mg) & Long half-life drug $(10)$ \\
\hline $\begin{array}{l}\text { Clinical visit record } \\
\text { per subject }(n)\end{array}$ & I8 (2-43 records) \\
\hline Time of dose administration & $\begin{array}{l}\text { Actual: MEMS cap opening time } \\
\text { Nominal: } 9 \text { pm (normal distribution, } \\
\text { SD = I hour) }\end{array}$ \\
\hline $\begin{array}{l}\text { Pharmacokinetic } \\
\text { sampling time }\end{array}$ & $\begin{array}{l}\text { Actual: } 8 \text { am to } 6 \mathrm{pm} \text { (uniform } \\
\text { distribution) } \\
\text { Nominal: Actual sampling time }+ \\
\text { reported time error (normal distribution, } \\
\mathrm{SD}=15 \text { minutes) }\end{array}$ \\
\hline Adherence rate (\%) & $\begin{array}{l}\text { Continuous adherence rate: } 7 \text {-day and } \\
\text { 2-day actual rate } \\
\text { Categorical adherence: Adherence rate } \\
\text { groups (very high, high, intermediate, low } \\
\text { and extremely low) based on weekly and } \\
\text { 2-day pattern }\end{array}$ \\
\hline Simulation replicates & 100 \\
\hline Simulation conditions & $\begin{array}{l}\text { MEMS data (number of doses taken) } \\
\text { Actual dosage time and actual } \\
\text { pharmacokinetic sampling time }\end{array}$ \\
\hline $\begin{array}{l}\text { Estimation conditions } \\
\text { for known dosage history }\end{array}$ & $\begin{array}{l}\text { Dosing: MEMS dosing (number of doses } \\
\text { taken) } \\
\text { Actual dosage time and actual sampling } \\
\text { time }\end{array}$ \\
\hline $\begin{array}{l}\text { Estimation conditions for } \\
\text { unknown dosing history }\end{array}$ & $\begin{array}{l}\text { I00\% adherence rate (all doses taken) } \\
\text { Nominal dosage time and reported } \\
\text { sampling time }\end{array}$ \\
\hline
\end{tabular}
half-life drug

Abbreviations: SD, standard deviation; MEMS, Medication Event Monitoring System. 
Simulated datasets, comprising "virtual subjects" with unique virtual concentration time profiles (ie, the virtual Cobs values) were generated using the sampling conditions outlined in the phenotypes study as well as the residual unknown variability in the prior pharmacokinetic model. The actual pharmacokinetic sampling time at each clinic visit was simulated to occur between 8 am to $6 \mathrm{pm}$ (clinic hours) using a pseudorandom uniform distribution. These simulated datasets provided individual pharmacokinetic parameters and concentration measurements (Cobs) for each virtual subject.

Simulation (Step 4.1) was also performed to create the subject with the incorrectly reported dosage history (nominal dose and dose-taking time) and the recorded pharmacokinetic sampling time. The erroneous nominal time of dose taken (incorrect dosing) was simulated from a normal distribution with a mean time of $9 \mathrm{pm}$, and a standard deviation (SD) of one hour. The reported pharmacokinetic sampling time was generated assuming a normal distribution using the actual time as the mean and a SD of 15 minutes. The pharmacokinetic samples were assumed to be taken at each clinic visit during regular clinic hours. An adherence rate of $100 \%$ was assumed for the unknown dosing history scenario.

Estimation (Step 3 and Step 4.2) was conducted under two conditions, ie, known dosage history, given the correct dosage history (MEMS cap data) and actual sampling time; and unknown dosage history, given incorrect (nominal) dosage history and reported (erroneous) sampling time. Population and individual pharmacokinetic parameters were estimated using NONMEM, and model predicted concentrations (Cpred and Cipred) were derived. The "observed" (ie, simulated from the virtual individuals as described in Step 2) concentrations were predicted based on individual-specific pharmacokinetic parameter estimates, and the Cpred/Cobs and Cipred/Cobs ratios were calculated in relation to the dosing history.

Due to the large number of simulation scenarios, the estimation of population and individual specific pharmacokinetic parameters, as well as the population predicted and individual Bayesian predicted concentrations, were obtained using the first-order method with the post hoc option in NONMEM. ${ }^{13}$

\section{Evaluation of robustness of Cipred/Cobs: Cpred/Cobs for true adherence prediction} Ratios with true adherence rates for 2- and 7-day windows

The distribution of Cpred/Cobs and Cipred/Cobs ratios were evaluated across all adherence patterns. Box and Whisker plots were generated for all the adherence rate categories. The box plot allows examination of the consistency of the Cpred/Cobs ratio in reflecting erratic adherence patterns (extremely low to high adherence). Under ideal conditions (known dosing history), the ratio of predicted and observed concentrations should be equal to one. Under the unknown dosing history scenario, concentrations were expected to be overpredicted if the adherence was less than $100 \%$ and underpredicted if the actual adherence was over $100 \%$. Thus, the systematic deviations of differences between the observed and the predicted concentrations could be reflected by the shift of the median ratio value with change of adherence rate.

\section{Rate classification using the established relationship between adherence rate and ratio}

The central tendency of the ratio (mean) at each adherence rate was calculated under unknown dosing history. Exponential decay models were applied to characterize the relationship between Cpred/Cobs, Cipred/Cobs, and actual adherence rate for the 7-day and 2-day time windows using NONMEM. The established relationships were then applied to predict adherence using the observed ratio. The ability to predict adherence correctly was used to evaluate the performance of the relationship between ratio and adherence rate.

The predicted adherence rate was classified based on minimum Euclidean distance classification criteria. ${ }^{17}$ At a given Cipred/Cobs ratio, the distance between the predicted rate and the observed rate was calculated using the equation:

$$
\mathrm{d}=\mid \text { Adherence }_{\text {pred }}-\text { Adherence }_{\text {obs }} \mid
$$

The predicted adherence rate was assigned to the class (observed rate) for which the distance "d" was the minimum. The percentage of the correctly assigned rates was calculated for the 7-day and 2-day adherence periods.

\section{Results Subjects and MEMS data}

MEMS data for a total of 65 patients were available from the ongoing depression phenotypes clinical trial during the first 6 months, providing 863 clinic visit records. The adherence rate was calculated for each clinic visit event (pharmacokinetic sampling). These rates were then grouped into different time windows, eg, adherence rates 7 days prior to a clinic visit and 2 days prior to a clinic visit. The adherence rates were found to be highly variable within subjects during the treatment period. The 7-day adherence pattern had 9.7\% 
of the events with more than 7 doses taken (rate $>100 \%$ ), $52.5 \%$ of events with $6-7$ doses taken (rate $85 \%-100 \%$ ), $19.0 \%$ of events with $3-5$ doses taken (rate $30 \%-85 \%$ ), $4.3 \%$ of events with 1-2 doses taken (rate $0 \%-30 \%$ ), and $14.5 \%$ with 0 doses taken (rate 0 ).

\section{Simulation and estimation results}

Box plots were generated for the long half-life drug under unknown dosing history and known dosage history scenarios (Figures 2 and 3). The known dosage history, where the estimation was based on the correct dosing history, had log median values of $\mathrm{Cpred} / \mathrm{Cobs}$ and Cipred/Cobs ratios that were approximately zero at each adherence rate. This is shown in Figure 2A and 2B.

Under unknown dosing history scenario, the estimation was based on the subjects' incorrect dosage history. The median of the Cpred/Cobs and Cipred/Cobs ratios increased with decreasing adherence rate. This is shown in Figure 2C and 2D. The differences of the Cipred/Cobs ratio across adherence rates appeared to be more substantial than that of Cpred/Cobs ratio under an unknown dosing history scenario.

\section{Rate classification using modeled relationship between rate and adherence ratio}

\section{Relationship between 7-day adherence rate and ratio}

The relationship between the median value of Cipred/Cobs ratio and the observed 7-day adherence rate was modeled. A biexponential function adequately described the relationship between Cipred/Cobs ratio and the weekly adherence rate. The observed adherence rate versus the Cipred/Cobs ratio, along with the Cipred from the unknown dosing history scenario, is shown in Figure 3. The exponential relationship between the Cipred/Cobs ratio and adherence rate is shown below:

Adherence $=699 e^{-3.15 \times \text { Cipred/Cobs }}+117 e^{-0.507 \times \text { Cipred/Cobs }}$

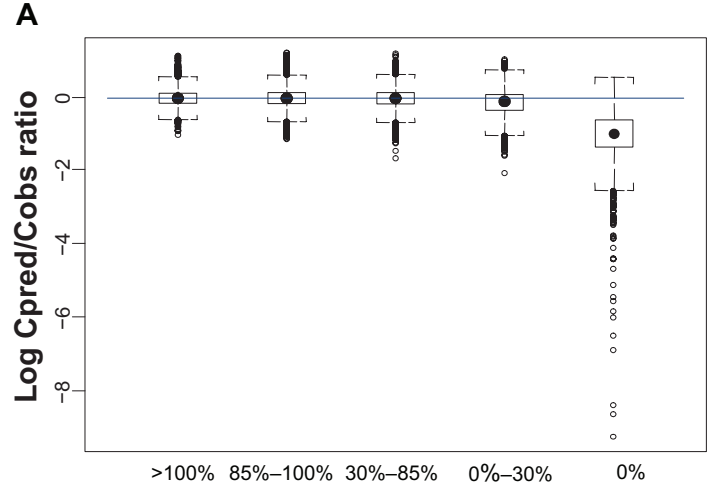

Weekly adherence rate pattern

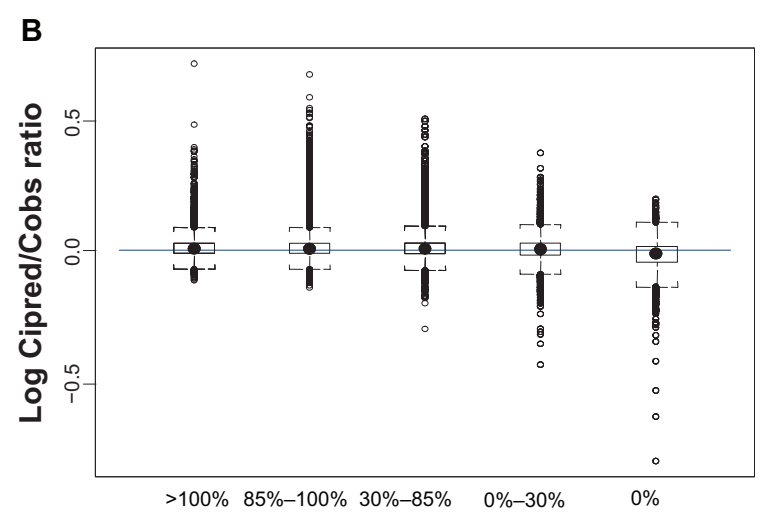

Weekly adherence rate pattern
C

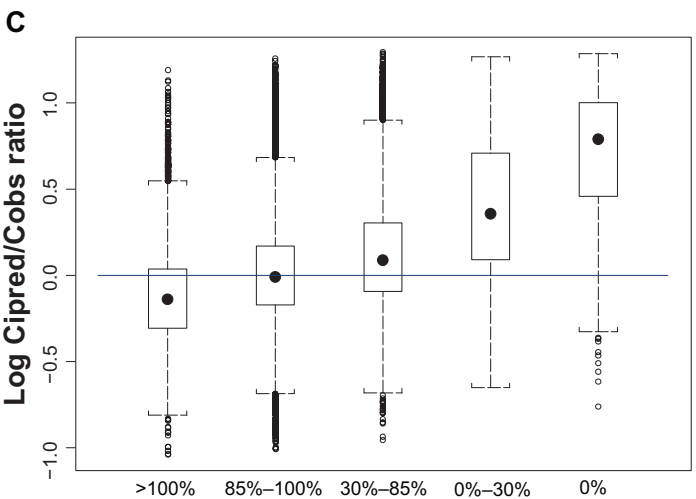

Weekly adherence rate pattern

D

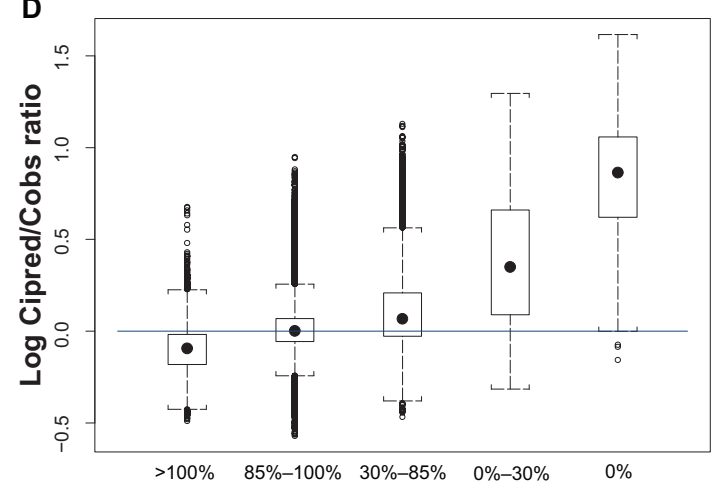

Weekly adherence rate pattern

Figure 2 Box plot of the ratio distribution at each adherence rate condition for a long half-life drug. The box plots represent median (dots), 25 th and 75 percentiles of the distribution. Notches show approximate $95 \%$ confidence limits for the median. A) Box-plot of the log Cpred:Cobs ratio under known dosage history. B) Box plot of the log Cipred:Cobs ratio under known dosage history. C) Box plot of the log Cpred:Cobs ratio under unknown dosage history. D) Box plot of the log Cipred:Cobs ratio under unknown dosage history.

Abbreviations: Cipred, individual predicted concentration; Cobs, observed concentration. 


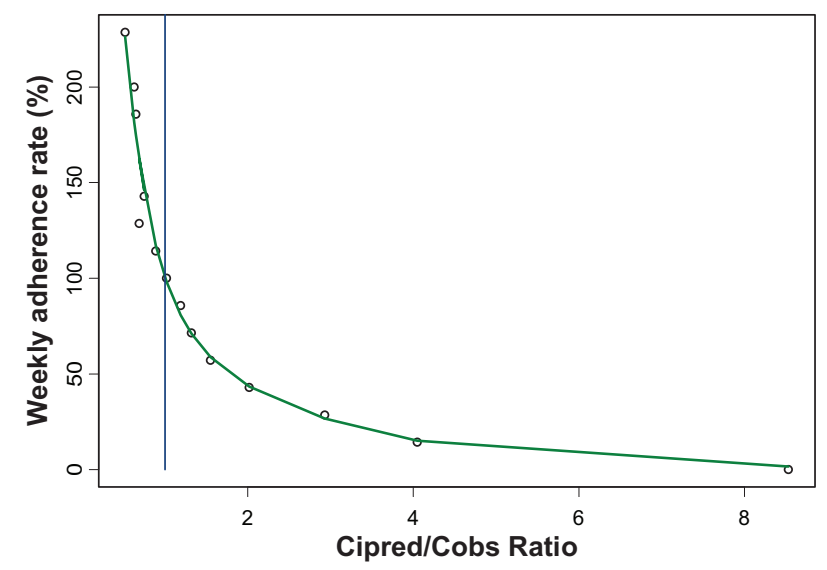

Figure 3 Association between Cipred:Cobs ratio and weekly adherence rate. Dots represented the median values of Cipred:Cobs ratio at each observed adherence rate condition. The line represented the model predicted weekly adherence rate at given Cipred:Cobs ratios. The vertical line represents a Cipred:Cobs ratio $=\mathrm{I}$ at a 100\% weekly adherence rate.

Abbreviations: Cipred, individual predicted concentration; Cobs, observed concentration.

\section{Relationship between 2-day adherence rate and ratio}

This same assessment was conducted for adherence rate categories arising for the 2-day period immediately prior to a clinic visit and concentration sampling. The relationship between this 2-day-based adherence rate and the Cipred/Cobs ratio was adequately described using a monoexponential function, as shown below:

$$
\text { Rate }=834 e^{-2.05 \times \text { Cipred/Cobs }}
$$

Similar relationships were found for the Cpred/Cobs ratio and adherence rate.

\section{Performance of ratio as a measure of adherence rate}

Rate classification for 7-day adherence rate

The exponential relationship developed above was applied for adherence rate prediction at each Cipred/Cobs ratio obtained under the unknown dosing history scenario, and then classified into the 7-day adherence category. The predicted rate classification was based on the minimum Euclidean distance classification criteria. ${ }^{17}$ The assigned rates were grouped based on weekly adherence patterns as described in the Methods section. The results of the correct rate classifications for each rate group are shown in Table 4 . The result of the 7-day adherence rate conditions showed that the correct assigned rate in pharmacokinetic samples measured under the high adherence rate condition (ie, hypercompliant) was $73.8 \%$. In pharmacokinetic samples measured under extremely low adherence rates $(0 \%$ in the last week) the percentage of instances correctly classified was $64.0 \%$. The rates were
Table 4 Correct classified 7-day adherence rate using Cipred: Cobs ratio as predictor

\begin{tabular}{lll}
\hline $\begin{array}{l}\text { Adherence rate } \\
\text { pattern }\end{array}$ & Events (n) & $\begin{array}{l}\text { Correct } \\
\text { classification (\%) }\end{array}$ \\
\hline Group 1 & 8014 & 73.82 \\
Group 2 & 45319 & 39.19 \\
Group 3 & 15972 & 36.65 \\
Group 4 & 2733 & 25.72 \\
Group 5 & 1128 & 64.01 \\
\hline
\end{tabular}

Notes: Group I, very high adherence rate condition (>100\%); Group 2, high adherence rate condition (85\%-100\%); Group 3, intermediate adherence rate condition (30\%-85\%); Group 4, low adherence rate condition ( $0 \%-30 \%)$; and Group 5, extremely low adherence rate condition $(0 \%)$.

Abbreviations: Cipred, individual predicted concentration; Cobs, observed concentration.

better classified in pharmacokinetic samples measured under extremely low $(0 \%)$ and extremely high adherence rate conditions $(>100 \%)$ than at intermediate adherence rate conditions.

Using Cipred/Cobs ratios to predict the 7-day adherence rate pattern, the overall rate of correct adherence classification was $42.3 \%$. The overall rate of correct adherence classification was $26.4 \%$ when the Cpred/Cobs ratio was used as the predictor.

\section{Rate classification for 2-day adherence rate}

The adherence condition was well classified for the event under extremely high (rate $>100 \%$ ) and extremely low (rate $=0 \%)$ adherence conditions. The correct classification rate (Table 5) was $80.8 \%$ for adherence $>100 \%$. The correct classification rate was $87.6 \%$ for adherence $=0 \%$.

Using the Cipred/Cobs ratios to predict the 2-day adherence rate pattern, the overall rate of correct adherence classification was $50.2 \%$. The overall rate of correct adherence classification was $29.9 \%$ using the Cpred/Cobs ratio as the predictor.

\section{Discussion}

It has been suggested that the inconsistency of drug exposure caused by variable adherence to prescribed therapy is the single

Table 5 Correct classified 2-day adherence rates using Cipred: Cobs ratio as predictor

\begin{tabular}{lll}
\hline $\begin{array}{l}\text { Adherence rate } \\
\text { pattern }\end{array}$ & Events (n) & $\begin{array}{l}\text { Correct } \\
\text { classification (\%) }\end{array}$ \\
\hline Group I & 8437 & 80.75 \\
Group 2 & 43653 & 44.69 \\
Group 3 & 17396 & 41.25 \\
Group 4 & 3680 & 87.58 \\
\hline
\end{tabular}

Notes: Group I, extremely high adherence rate condition (>100\%); Group 2, high adherence rate condition (100\%); Group 3, intermediate adherence rate condition (50\%); Group 4, extremely low adherence rate condition ( $0 \%$ ).

Abbreviations: Cipred, individual predicted concentration; Cobs, observed concentration. 
largest source of variance in the drug response. ${ }^{11}$ Therefore, adherence plays an important role in pharmacotherapy efficacy assessment, because the dosage adjustments may not be relevant if the subject is inconsistently receiving the prescribed medicine. The aim of this study was to identify erratic adherence using a modeling and simulation approach based on the possibility of taking a single concentration measure from a virtual patient and assessing whether or not this single measure provides an insight into how well the patient adhered with their medication regimen. This is the first study to evaluate the performance of the deviation between the population pharmacokinetic model predicted versus observed concentrations as an indicator of adherence rate for a target population. This was accomplished by developing a specific parametric relationship between the adherence rate calculated from MEMS data and the ratio of Cipred/Cobs and Cpred/Cobs as metrics reflecting these differences.

Seven-day and 2-day time windows were selected as two extreme conditions for adherence rate calculation. These windows were selected to link closely the information on dose taken with inconsistency of drug exposure. Our simulation study suggested that the population pharmacokinetic modeling approach was most useful in the detection of extremely high and extremely low adherence rate patterns (Tables 4 and 5). These results are not unexpected, given that intermediate adherence rate patterns can arise from many different dosing histories, resulting in false assignment based on the ratio. Consider the following example using the 2-day adherence rate pattern. Subject A has taken the dose on the first day of the 2-day time window, subject $\mathrm{B}$ has taken the dose on the second day of the 2-day time window, and subject $\mathrm{C}$ takes all doses correctly in the 2-day time window. Subjects A and B have a 50\% adherence rate, but their Cipred/Cobs ratios will be different. This explains how the Cpred/Cobs and Cipred/Cobs ratios predicts adherence rates reasonably well only at the extremes (eg, $0 \%$ and $>100 \%$ adherence).

The 2-day adherence rate patterns had a higher percentage of correct assignment than did 7-day adherence patterns (Tables 4 and 5). One explanation for this different percentage of correct assignment is the result of different time windows used for adherence rate calculation, as discussed above. Specifically, for the 2-day adherence rate pattern, if the adherence rate is $50 \%$ (one dose taken in the 2-day time window), there will be two combinations of how this one dose is actually taken, whereas for the 7-day adherence rate pattern, if the adherence rate is $14 \%$ (one dose taken in the 7-day time window), there are seven possible ways this one dose is actually taken. Therefore, the ratio metric and the 2-day adherence rate will have a stronger association than the association between the ratio and 7-day adherence rate.

Only $20 \%$ of the high, intermediate, and low adherence rates were correctly assigned. Therefore, electronic monitoring, in addition to population pharmacokinetics, is necessary under these conditions, because these types of inconsistencies may still contribute adverse drug reactions, as well as treatment resistance, and are not detectable using a Cipred/Cobs or Cpred/Cobs ratio approach. Thus, the single deviation observed using the population pharmacokinetic methodology has some serious limitations. The use of multiple observations (ie, as in the Brundage study) ${ }^{18}$ may be necessary to improve adherence classification or link these deviations to response. These results identified that use of MEMS dosing history monitoring in everyday clinical practice combined with sparse pharmacokinetic sampling could be a more reliable approach.

The delayed effect between pharmacokinetic and pharmacodynamic activity is common with central nerve system compounds, due to their mechanism of action in that most central nerve system compounds target neurotransmitters in the brain neuron synapse. The limitation of paper is that it only evaluated the impact of nonadherence on pharmacokinetics and the performance of Cipred/Cobs as a measure of erratic drug exposure, and the impact of erratic drug exposure on pharmacodynamics was not investigated in this simulation.

\section{Conclusion}

This simulation study demonstrated that the combination of the population pharmacokinetic model in the absence of robust adherence information from a source such as MEMS caps is limited as a method to detect erratic exposure with specific exceptions, eg, a subject has taken virtually no drug (extremely low adherence condition) or more drug than prescribed (the extremely high adherence condition). Both the extremely high and extremely low adherence rate conditions are reasonably well predicted by the Cipred/Cobs and Cpred/Cobs ratio with a single observation. If the adherence patterns reflect an intermediate number of dosages taken, the single Cpred/Cobs or Cipred/Cobs measurement does not adequately reflect the adherence in this range. In this case, MEMS information in conjunction with population pharmacokinetics may be necessary. Therefore, this ratio may be helpful in identifying those who took virtually no drug or took more than the prescribed drug regimen. 


\section{Acknowledgment}

This work was supported by grants from the National Institute for Biomedical Imaging and Bioengineering, Advanced Center for Interventions and Services Research in Late Life, the Metrum Institute, and the Indiana Clinical and Translational Sciences Institute.

\section{Disclosure}

Dr. Pollock receives research support from the National Institute of Health and the Canadian Institutes of Health Research. Within the past five years he has been a member of the advisory board of Lundbeck Canada (final meeting was May 2009) and Forest Laboratories (final meeting was March 2008). Dr. Pollock has served one time as a consultant for Wyeth (October 2008) and Takeda (July 2007). He was also a faculty member of the Lundbeck International Neuroscience Foundation (LINF) (final meeting was April 2010). Dr. Frank serves on an advisory board of Servier International and receives royalties from Guilford Press

\section{References}

1. Diaz E, Levine HB, Sullivan MC, et al. Use of the Medication Event Monitoring System to estimate medication compliance in patients with schizophrenia. J Psychiatry Neurosci. 2001;26:325-329.

2. Meijer WE, Bouvy ML, Heerdink ER, Urquhart J, Leufkens HG. Spontaneous lapses in dosing during chronic treatment with selective serotonin reuptake inhibitors. Br J Psychiatry. 2001;179:519-522.

3. Thompson C, Peveler RC, Stephenson D, McKendrick J. Compliance with antidepressant medication in the treatment of major depressive disorder in primary care: A randomized comparison of fluoxetine and a tricyclic antidepressant. Am J Psychiatry. 2000;157:338-343.

4. Kruse W, Weber E. Dynamics of drug regimen compliance - its assessment by microprocessor-based monitoring. Eur J Clin Pharmacol. 1990;38:561-565.

5. Urquhart J. Role of patient compliance in clinical pharmacokinetics A review of recent research. Clin Pharmacokinet. 1994;27:202-215.
6. Urquhart J. Compliance and clinical trials. Lancet. 1991;337: 1224-1225.

7. Urquhart J. The impact of compliance on drug development. Transplant Proc. 1999;31:39S.

8. Urquhart J. Introduction to pharmionics: The vagaries of ambulatory patients adherence to prescribed drug dosing regimens and some of their clinical and economic consequences. In: Mann RD, Andrews EB, editors. Pharmacovigilance. 2nd ed. Chichester, UK: John Wiley \& Sons Ltd; 2007.

9. Urquhart J, Bernard V. Taxonomy of patient compliance-related events in drug trials. National Cancer Institute Conference on Causal Inference, 2001 Aug 9-13; Snowbird, UT, US.

10. Vrijens B, Tousset E, Rode R, Bertz R, Mayer S, Urquhart J. Successful projection of the time course of drug concentration in plasma during a 1-year period from electronically compiled dosing-time data used as input to individually parameterized pharmacokinetic models. J Clin Pharmacol. 2005;45:461-467.

11. Vrijens B, Gross R, Urquhart J. The odds that clinically unrecognized poor or partial adherence confuses population pharmacokinetic/ pharmacodynamic analyses. Basic Clin Pharmacol Toxicol. 2005;96: 225-227.

12. Frank E, Cassano GB, Rucci P, et al. Addressing the challenges of a cross-national investigation: Lessons from the Pittsburgh-Pisa study of treatment-relevant phenotypes of unipolar depression. Clin Trials. 2008;5:253-261.

13. Beal B, Sheiner L. NONMEM user's guide, Part I. San Francisco, CA: University of California at San Francisco; 1992.

14. Sheiner LB, Rosenberg B, Marathe VV. Estimation of population characteristics of pharmacokinetic parameters from routine clinical data. J Pharmacokinet Biopharm. 1977;5:445-479.

15. Sogaard B, Mengel H, Rao N, Larsen F. The pharmacokinetics of escitalopram after oral and intravenous administration of single and multiple doses to healthy subjects. J Clin Pharmacol. 2005;45:1400-1406.

16. Gutierrez MM, Rosenberg J, Abramowitz W. An evaluation of the potential for pharmacokinetic interaction between escitalopram and the cytochrome P450 3A4 inhibitor ritonavir. Clin Ther. 2003;25: 1200-1210.

17. Weisstein EW. Distance. From MathWorld-A WolframMathWorld Web Resource: http://mathworld.wolfram.com/Distance.html. Accessed Feb 2011.

18. Brundage RC, Yong FH, Fenton T, Spector SA, Starr SE, Fletcher CV. Intrapatient variability of efavirenz concentrations as a predictor of virologic response to antiretroviral therapy. Antimicrob Agents Chemother. 2004;48:979-984.
Neuropsychiatric Disease and Treatment

\section{Publish your work in this journal}

Neuropsychiatric Disease and Treatment is an international, peerreviewed journal of clinical therapeutics and pharmacology focusing on concise rapid reporting of clinical or pre-clinical studies on a range of neuropsychiatric and neurological disorders. This journal is indexed on PubMed Central, the 'PsycINFO' database and CAS, and is the official

\section{Dovepress}

journal of The International Neuropsychiatric Association (INA). The manuscript management system is completely online and includes a very quick and fair peer-review system, which is all easy to use. Visit http://www.dovepress.com/testimonials.php to read real quotes from published authors. 